\title{
Soluções atrativas para o monitoramento populacional de Zaprionus indianus (Diptera: Drosophilidae) em pomar de figo ${ }^{1}$
}

\author{
Mauricio Paulo Batistella Pasini², Dionisio Link ${ }^{3}$, Alessandro Dal'Col Lúcio², Andriéle Taciane Wansing ${ }^{4}$
}

\begin{abstract}
Attractive solutions for monitoring Zaprionus indianus

(Diptera: Drosophilidae) populations in fig orchard

The fig fly (Zaprionus indianus) is one of the major fig pests in Brazil, being its monitoring important for reducing the use of chemical insecticides. This study aimed at evaluating the efficiency of attractive food for Z. indianus, in fig orchard. The experiment was installed in a domestic fig ('Roxo de Valinhos' cultivar) orchard, using five treatments (fig juice at $50 \%$, sugarcane molasses at $7 \%$ and grape juice at $30 \%, 40 \%$ and $50 \%$ ), with five replicates, in a completely randomized design. A total of 3,284 adult fig flies (1,983 female and 1,301 male) were captured, representing $42 \%$ of the total insects and $62 \%$ of the drosophilids captured. The proportion of males and females was similar for all attractive solutions. Fig juice at 50\% was effective in capturing the fig fly, being recommended for its monitoring in domestic orchards. Sugarcane molasses at $7 \%$, despite its effectiveness, did not present regularity in fig fly capturing, being only indicated for periods of less than 28 days. At the concentrations tested, the grape juice was not effective.
\end{abstract}

KEY-WORDS: Ficus carica L.; insect-pest; fig fly.

\section{INTRODUÇÃO}

A figueira (Ficus carica L.) é uma das mais antigas frutíferas cultivadas no mundo (Silva et al. 2010). Dentre diversas cultivares, a Roxo de Valinhos é a mais plantada no Brasil, e seus frutos podem ser utilizados tanto para consumo in natura, como para a indústria (Silva et al. 2011).

A mosca-do-figo, Zaprionus indianus (Gupta, 1970) (Diptera: Drosophilidae), é uma das principais pragas da cultura do figo, sendo característica de regiões tropicais (Vilela et al. 2000, Pasini \& Link 2011). Esta praga foi, inicialmente, detectada no Estado de

\section{RESUMO}

A mosca-do-figo (Zaprionus indianus) é uma das principais pragas da cultura do figo, no Brasil, sendo o seu monitoramento importante para a redução no uso de inseticidas químicos. Este estudo objetivou avaliar a eficiência de atrativos alimentares para o monitoramento de Z. indianus, em pomar de figo. O experimento foi instalado em pomar doméstico de figo (cv. Roxo de Valinhos), utilizando-se cinco tratamentos (suco de figo a $50 \%$, melaço de cana a $7 \%$ e suco de uva a $30 \%, 40 \%$ e $50 \%$ ), com cinco repetições, em delineamento inteiramente casualizado. Foram capturados 3.284 indivíduos adultos da mosca-do-figo (1.983 fêmeas e 1.301 machos), representando $42 \%$ do total de insetos e $62 \%$ dos drosofilídeos capturados. A proporção de machos e fêmeas foi similar em todas as soluções atrativas. A solução suco de figo a 50\% foi eficiente na captura da moscado-figo, podendo ser recomendada para o seu monitoramento em pomares domésticos. A solução melaço de cana a 7\%, embora eficiente, não apresentou regularidade na captura da mosca-dofigo, sendo indicada apenas para períodos inferiores a 28 dias. Nas concentrações testadas, o suco de uva não foi eficiente.

PALAVRAS-CHAVE: Ficus carica L.; inseto-praga; moscado-figo.

São Paulo, em 1999, e fatores como condições ambientais favoráveis, elevado número de plantas hospedeiras (74 espécies de 31 famílias), ampla adaptabilidade, ciclo de desenvolvimento curto e alto potencial biótico foram fundamentais para a sua dispersão pelo território brasileiro (Vilela et al. 2000, Pires et al. 2008, Galego \& Careto 2010, Mata et al. 2010, Commar et al. 2012).

Ao atacar a cultura do figo, a mosca deposita seus ovos no ostíolo, na fase de amadurecimento, e as larvas penetram no fruto, tornando-o impróprio para o consumo, causando o seu apodrecimento e inutilizando-o comercialmente (Stein et al. 2003, Pasini et al. 2011a).

1. Trabalho recebido em mar./2013 e aceito para publicação em ago./2013 ( $\mathrm{n}^{\circ}$ registro: PAT 22888).

2. Universidade Federal de Santa Maria (UFSM), Centro de Ciências Rurais, Santa Maria, RS, Brasil. E-mails: mauricio.pasini@gmail.com, adlucio@ufsm.br.

3. In Memoriam

4. Centro Universitário Franciscano (Unifra), Santa Maria, RS, Brasil.E-mail: andriele.tw@gmail.com. 
A eficiência no monitoramento da mosca-do-figo é dependente da disponibilidade de bons atrativos alimentares. A busca por sistemas eficientes de monitoramento é prioridade para o manejo da praga, tornando-se base para ações de prevenção e controle (Raga et al. 2006, Malo et al. 2012).

Trabalhos recentes indicam que o uso de soluções atrativas, em armadilhas frasco caça-mosca, pode ser uma alternativa de baixo custo e viável para a ficicultura no Brasil (Pasini et al. 2011a, 2011b, 2012). Formas de controle e recomendações de manejo para esta praga têm sido propostas, sendo algumas eficientes, porém, de baixa funcionalidade, requerendo tempo e custo para suas implementações (Pasini et al. 2011a).

O uso de frascos caça-mosca oriundos da reciclagem de materiais provenientes de propriedades rurais e de atrativos alimentares confeccionados a partir de frutas, restos culturais e alimentares torna-se a base para ações supressivas, como opção eficiente e de baixo custo, contribuindo para a redução no uso de agrotóxicos (Pasini et al. 2012). Outro fator relevante é a necessidade de desenvolvimento de novas soluções atrativas, que possam ser alternativas eficientes em períodos de escassez de matéria-prima, além da comparação entre as soluções existentes, em relação à eficiência de atratividade (Pasini et al. 2011b).

Os consumidores estão cada vez mais exigentes em suas demandas por produtos menos agressivos ao meio ambiente e, principalmente, com menos riscos à saúde humana (Fornazier \& Waquil 2011). A implementação de soluções atrativas, além de proporcionar redução no uso de pesticidas, torna-se uma alternativa para a produção de figos orgânicos, visto serem poucas as opções para manejo e controle fitossanitário, principalmente de pragas, para este modo de produção (Mazor 2009, Epsky et al. 2011, Teixeira et al. 2011).

Assim, este estudo objetivou avaliar a eficiência de diferentes atrativos alimentares, para fins de monitoramento de Z. indianus, em pomar de figo.

\section{MATERIAL E MÉTODOS}

O experimento foi instalado em pomar doméstico de figo da cultivar Roxo de Valinhos, no município de Agudo, RS (latitude: -29,653844; longitude: -53,236496), cuja área total de figueiras é de $300 \mathrm{~m}^{2}$. Com base na classificação de Köppen, o clima da região é do tipo Cfa, subtropical úmido, sem estação seca e com verões quentes (Heldwein et al. 2009). O pomar está plantado com espaçamento de $2,0 \mathrm{~m} \times 2,5 \mathrm{~m}$ e solo coberto por vegetação rasteira.

As plantas utilizadas no experimento estavam no início da produção, com presença da mosca-do-figo constatada e identificada na área por monitoramento, por meio de armadilhas e recolhimento de frutos. Para a confirmação da espécie, os indivíduos capturados foram identificados de acordo com chave proposta por Yassin \& David (2010). Não houve aplicação de agroquímicos, durante a execução da pesquisa.

Foram utilizados cinco tratamentos, correspondendo aos seguintes atrativos nas armadilhas e suas respectivas concentrações: FA - suco de figo a 50\% (100 mL de suco de figo $+100 \mathrm{~mL}$ de água), padrão 1 (Pasini et al. 2011a, 2011b); MA - melaço de cana a 7\% (14 mL de melaço de cana-de-açúcar $+186 \mathrm{~mL}$ de água), padrão 2 (Raga et al. 2006); SUA1 - suco de uva a $30 \%(60 \mathrm{~mL}$ de suco de uva $+140 \mathrm{~mL}$ de água); SUA2 - suco de uva a 40\% (80 mL de suco de uva + $120 \mathrm{~mL}$ de água); e SUA3 - suco de uva a $50 \%$ (100 mL de suco de uva $+100 \mathrm{~mL}$ de água), com cinco repetições cada, totalizando 25 unidades experimentais, distribuídas em delineamento inteiramente casualizado, correspondendo, para cada unidade experimental, a um frasco caça-mosca.

$\mathrm{O}$ ensaio foi instalado em 21 de janeiro de 2011 e conduzido por oito semanas, divididas em dois subperíodos de quatro semanas, correspondentes ao período de permanência da solução atrativa na armadilha (28 dias). Durante o subperíodo, o volume de atrativo não foi completado, devido à baixa evaporação da solução atrativa, no tipo de armadilha utilizado (Pasini \& Link 2011).

Os sucos de figo e uva foram extraídos de frutas oriundas da propriedade onde se instalou o experimento e elaborados de acordo com Pasini et al. (2011a). Para cada armadilha frasco caça-mosca, constituída de garrafa Pet de $600 \mathrm{~mL}$ incolor, oriunda de reciclagem, foram utilizados $200 \mathrm{~mL}$ de solução atrativa. Em cada garrafa, foram feitas duas perfurações de $8 \mathrm{~mm}$ de diâmetro, para a entrada dos insetos (Pasini \& Link 2011). As armadilhas foram dispostas na face oeste do dossel da planta, ao abrigo da luz solar, a $0,50 \mathrm{~m}$ do solo, sendo mantidas fixas as suas posições, durante o período do estudo.

Semanalmente, foram retiradas as 25 amostras contendo os insetos capturados em cada unidade experimental. Estes foram separados da solução 
atrativa, com o auxílio de uma peneira, e o líquido remanescente foi reposto na armadilha. As amostras foram levadas para o laboratório, onde foram triadas, e os adultos da mosca-do-figo sexados e identificados (Linde 2010, Yassin \& David 2010).

Os valores de mosca por armadilha por dia (MAD), obtidos nos diferentes tratamentos, em cada avaliação, foram calculados com base na média de captura das quatro semanas de avaliação, dividida pelo período entre as avaliações, perfazendo sete dias. Os valores de MAD foram organizados em fatorial $5 \times 2$ (cinco tratamentos $\mathrm{x}$ dois subperíodos de 28 dias, correspondentes ao período de permanência da solução atrativa na armadilha), com cinco repetições. Posteriormente, foram submetidos à avaliação da normalidade dos erros, pelo teste de Anderson Darling, e, em seguida, submetidos à transformação $\sqrt{\mathrm{x}}+1$, análise de variância (Anova) e comparação das médias, pelo teste Tukey.

Para as comparações de médias semanais, foi aplicado o método de análise estatística por contrastes ortogonais, e a significância de cada contraste foi avaliada pelo teste F (Gomez \& Gomez 1984). Os quatro contrastes para o experimento, entre os níveis dos tratamentos avaliados dentro de cada data de avaliação, foram: C1 = 4FA - MA - SU1 - SU2 - SU3 (comparação entre o padrão 1 e os demais tratamentos); C2 = 3MA - SU1 - SU2 - SU3 (comparação entre o padrão 2 e as soluções à base de suco de uva); C3 = 2SU3 - SU1 - SU2 (comparação entre as concentrações de suco de uva a $50 \%$ e a $30 \%$ e $40 \%$ ); e C4 = SU2 - SU1 (comparação entre as concentrações de suco de uva a $40 \%$ e a $30 \%$ ).

Para todas as análises estatísticas, feitas com o auxílio do programa computacional Sisvar (Ferreira 2011), adotou-se 5\% de probabilidade de erro.

\section{RESULTADOS E DISCUSSÃO}

Foram capturados 3.284 indivíduos adultos da mosca-do-figo (1.983 fêmeas e 1.301 machos), representando $42 \%$ do total de insetos e $62 \%$ dos drosofilídeos capturados pelas armadilhas. A proporção de machos e fêmeas foi similar à encontrada por Pasini et al. (2011a, 2011b).

A interação foi significativa entre os fatores estudados, indicando efeito do subperíodo de 28 dias sobre a captura da mosca-do-figo. As soluções atrativas suco de figo a $50 \%$ e suco de uva a $30 \%$, $40 \%$ e $50 \%$ apresentaram maior captura média, no primeiro subperíodo. Contudo, o melaço de cana a $7 \%$ apresentou comportamento contrário, com maior captura no segundo subperíodo (Tabela 1).

A solução atrativa suco de figo a $50 \%$ foi eficiente na atratividade, para a mosca-do-figo, diferindo, estatisticamente, das demais soluções, no primeiro subperíodo. Contudo, no segundo subperíodo, o suco de figo a $50 \%$ e o melaço de cana a $7 \%$ apresentaram maior eficiência na captura da mosca (Tabela 1).

A solução atrativa suco de figo a $50 \%$ capturou $39 \%$ do total de adultos de Z. indianus. Com desempenho semelhante, Pasini et al. (2011a, 2011b) obtiveram melhor eficiência com o atrativo suco de figo a $50 \%$. As soluções com suco de uva, nas concentrações de $30 \%, 40 \%$ e $50 \%$, obtiveram, respectivamente, $6 \%, 9 \%$ e $17 \%$ do total de adultos de $Z$. indianus capturados, havendo relação direta entre o aumento na concentração do atrativo e a captura de adultos da mosca-do-figo. Raga \& Souza Filho (2003) e Raga et al. (2006) obtiveram valores de captura superiores, para soluções atrativas à base de melaço, considerando seu uso adequado para a captura e monitoramento de $Z$. indianus.

Nos dois subperíodos, as soluções atrativas suco de uva a $30 \%$ e a $40 \%$ apresentaram desempenho inferior às demais (Tabela 1), sendo o seu uso não recomendável para o monitoramento da mosca-do-figo. Scoz et al. (2006) testaram suco de uva para a captura de moscas das frutas, em pomar de pêssego, obtendo valores intermediários para este atrativo, em

Tabela 1. Número médio de insetos capturados por armadilha por dia (MAD) \pm desvio padrão de Zaprionus indianus (Diptera: Drosophilidae), nos diferentes tratamentos e nos períodos relativos à permanência da solução atrativa, em pomar doméstico de figo cv. Roxo de Valinhos (Agudo, RS, 2011).

\begin{tabular}{ccc}
\hline \multirow{2}{*}{ Tratamento } & \multicolumn{2}{c}{ Subperíodo } \\
\cline { 2 - 3 } & Primeiro & Segundo \\
\hline FA $^{1}$ & $5,49 \pm 0,96 \mathrm{Aa}^{*}$ & $3,78 \pm 1,28 \mathrm{Ba}$ \\
MA & $1,96 \pm 0,93 \mathrm{Bc}$ & $4,74 \pm 2,64 \mathrm{Aa}$ \\
SU1 & $0,95 \pm 0,20 \mathrm{Ac}$ & $0,36 \pm 0,18 \mathrm{Bc}$ \\
SU2 & $1,63 \pm 1,08 \mathrm{Ac}$ & $0,48 \pm 0,19 \mathrm{Bc}$ \\
SU3 & $2,91 \pm 0,90 \mathrm{Ab}$ & $1,16 \pm 0,51 \mathrm{Bb}$ \\
\hline
\end{tabular}

${ }^{1}$ FA: suco de figo + água $(100 \mathrm{~mL}+100 \mathrm{~mL})$; MA: melaço + água $(14 \mathrm{~mL}+$ $186 \mathrm{~mL})$; SU1: suco de uva + água $(60 \mathrm{~mL}+140 \mathrm{~mL})$; SU2: suco de uva + água $(80 \mathrm{~mL}+120 \mathrm{~mL})$; SU3: suco de uva + água $(100 \mathrm{~mL}+100 \mathrm{~mL}) . *$ Médias seguidas por letras diferentes, dentro de cada subperíodo (linha, letras minúsculas) e entre os subperíodos (coluna, letras maiúsculas), diferem, estatisticamente, pelo teste Tukey, a $5 \%$. Valores médios transformados $(\sqrt{x}+1)$ e submetidos à análise de variância. 
comparação com a levedura Torula. No entanto, no primeiro subperíodo, a solução atrativa suco de uva a $50 \%$ apresentou desempenho superior à solução melaço de cana a 7\%. Contrariamente, o melaço de cana a $7 \%$ foi inferior no primeiro subperíodo e superior no segundo. Estes comportamentos podem estar relacionados à presença de frutos de figo em decomposição, nas proximidades das armadilhas, e/ ou à fermentação das soluções, havendo maior liberação de substâncias atrativas, em seus respectivos períodos de maior captura.

Pasini et al. (2011a) observaram que frutos em decomposição na planta, ou sobre o chão, servem como sítios de alimentação, cópula e desenvolvimento, para a mosca, além de atraírem seus adultos, o que contribui para o aumento na captura dos mesmos, pela armadilha mais próxima.

Dentre os contrastes analisados, $\mathrm{C} 1$ mostrou-se significativo (Tabela 2) em, praticamente, todas as avaliações, indicando maior regularidade e eficiência na captura de adultos da mosca-do-figo em suco de figo a 50\%, em relação aos demais tratamentos (Figura 1). Na $5^{\mathrm{a}}$ e $6^{\mathrm{a}}$ datas de avaliação, não houve significância no contraste de suco de figo a 50\%, com relação aos demais tratamentos, fato justificado pelos elevados valores médios de captura com melaço de cana a $7 \%$, nas respectivas datas.

No segundo contraste (C2), não houve significância na $1^{\mathrm{a}}, 2^{\mathrm{a}}$ e $3^{\mathrm{a}}$ datas de avaliação, fato justificado pelas elevadas médias de captura com suco de uva a $40 \%$ e a $50 \%$, nos respectivos períodos, com o aumento posterior no valor médio de captura. $\mathrm{O}$ melaço de cana a $7 \%$ diferiu, estatisticamente, das soluções com base em suco de uva. $\mathrm{Na} 5^{\mathrm{a}}$ e $6^{\mathrm{a}}$ semanas de captura, o melaço de cana a $7 \%$ apresentou médias superiores aos demais tratamentos (Figura 1). Este resultado demonstra que a presença de frutos em decomposição no pomar e em sua proximidade contribuiu para o desenvolvimento da mosca, sua disseminação e seu ataque sobre figos (Vilela et al. 2000, Pasini et al. 2011b). Embora tenha apresentado maior eficiência de captura em alguns períodos, o melaço de cana a $7 \%$ não apresentou comportamento regular, podendo sua utilização no monitoramento ser revista ou indicada para menores períodos (inferiores a 28 dias).

No terceiro contraste, o tratamento com suco de uva a $50 \%$ diferenciou-se, estatisticamente, na $3^{\text {a }}$ e $4^{\text {a }}$ datas de avaliação, sendo que, nas demais datas,

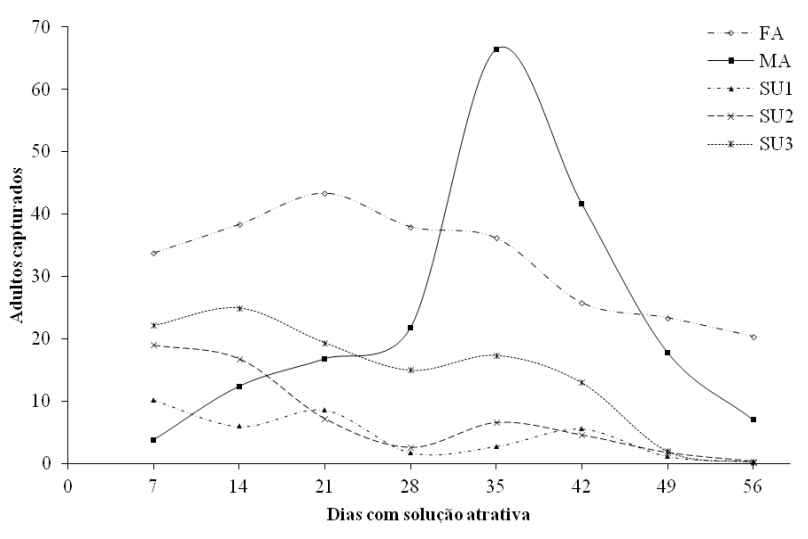

Figura 1. Variação no número de adultos de Zaprionus indianus (Diptera: Drosophilidae) capturados em diferentes dias com solução atrativa, em pomar doméstico de figo (Agudo, RS, 2011). FA: suco de figo + água (100 mL + $100 \mathrm{~mL})$; MA: melaço + água (14 mL + $186 \mathrm{~mL})$; SU1: suco de uva + água $(60 \mathrm{~mL}+140 \mathrm{~mL})$; SU2: suco de uva + água $(80 \mathrm{~mL}+120 \mathrm{~mL})$; SU3: suco de uva + água $(100 \mathrm{~mL}+100 \mathrm{~mL})$.

Tabela 2. Médias de captura de Zaprionus indianus (Diptera: Drosophilidae) e significância dos contrastes ortogonais, para soluções atrativas em pomar de figo (Agudo, RS, 2011).

\begin{tabular}{|c|c|c|c|c|c|c|c|c|c|}
\hline \multirow{2}{*}{ Data das avaliações } & \multicolumn{5}{|c|}{ Solução atrativa ${ }^{1}$} & \multicolumn{4}{|c|}{ Contraste ortogonal $^{2}$} \\
\hline & FA & MA & SU1 & SU2 & SU3 & $\mathrm{C} 1$ & $\mathrm{C} 2$ & $\mathrm{C} 3$ & $\mathrm{C} 4$ \\
\hline 21/jan & 33,8 & 3,8 & 10,2 & 19,0 & 22,2 & $*$ & ns & ns & ns \\
\hline 30/jan & 38,4 & 12,4 & 6,0 & 16,8 & 25,0 & $*$ & ns & ns & ns \\
\hline $06 / \mathrm{fev}$ & 43,4 & 16,8 & 8,6 & 7,2 & 19,4 & $*$ & ns & $*$ & ns \\
\hline $13 /$ fev & 38,0 & 21,8 & 1,8 & 2,6 & 15,0 & $*$ & $*$ & $*$ & ns \\
\hline $20 / \mathrm{fev}$ & 36,2 & 66,4 & 2,8 & 6,6 & 17,4 & ns & $*$ & ns & ns \\
\hline $27 / \mathrm{fev}$ & 25,8 & 41,6 & 5,6 & 4,6 & 13,0 & ns & $*$ & $\mathrm{~ns}$ & ns \\
\hline 02/mar & 23,4 & 17,8 & 1,2 & 1,8 & 2,0 & $*$ & $*$ & ns & ns \\
\hline 09/mar & 20,4 & 7,0 & 0,4 & 0,4 & 0,2 & $*$ & $*$ & ns & ns \\
\hline
\end{tabular}

${ }^{1} \mathrm{FA}$ : suco de figo + água $(100 \mathrm{~mL}+100 \mathrm{~mL})$; MA: melaço + água $(14 \mathrm{~mL}+186 \mathrm{~mL})$; SU1: suco de uva + água $(60 \mathrm{~mL}+140 \mathrm{~mL}) ; \mathrm{SU} 2: \mathrm{suco}$ de uva + água $(80 \mathrm{~mL}+$ $120 \mathrm{~mL})$; SU3: suco de uva + água $(100 \mathrm{~mL}+100 \mathrm{~mL}) .{ }^{2} \mathrm{C} 1:$ FFA - MA - SU1 - SU2 - SU3; C2: 3MA - SU1 - SU2 - SU3; C3: 2 SU3 - SU1 - SU2; C4: SU2 - SU1. * Significativo a $5 \%$, pelo teste F; ns: não significativo. 
não houve diferença entre as médias no contraste. Embora o suco de uva a 50\% apresentasse maior média de captura, em relação ao suco de uva a $40 \%$ e a $30 \%$, na maioria das datas de avaliação, esta solução apresentou, de modo geral, baixa eficiência, quando comparada ao suco de figo a $50 \%$ e melaço de cana a $7 \%$. O quarto contraste foi não significativo para todas as datas de avaliação.

A solução atrativa melaço de cana a $7 \%$ apresentou comportamento de captura diferente das demais soluções (Figura 1), com captura crescente até a $5^{\text {a }}$ semana e decrescente nas demais datas de avaliação. Os demais tratamentos apresentaram comportamento de captura similar ao do suco de figo a 50\%, análogo ao encontrado por Pasini et al. (2011a), indicando redução gradativa na população da mosca, decorrente da captura pelas armadilhas e menor número de frutos por planta.

\section{CONCLUSÕES}

1. O suco de figo a $50 \%$ foi eficiente na captura da mosca-do-figo, sendo recomendada a sua utilização para o monitoramento de Zaprionus indianus, em pomares domésticos.

2. O melaço de cana a 7\% não apresentou regularidade na captura da mosca-do-figo.

3. Nas concentrações testadas, o suco de uva não foi eficiente.

\section{REFERÊNCIAS}

COMMAR, L. S. et al. Taxonomic and evolutionary analysis of Zaprionus indianus and its colonization of Palearctic and Neotropical regions. Genetics and Molecular Biology, São Paulo, v. 35, n. 2, p. 395-406, 2012.

EPSKY, N. D. et al. Comparison of synthetic food-based lures and liquid protein baits for capture Anastrepha suspense (Diptera: Tephritidae) adults. Florida Entomologist, Gainesville, v. 94, n. 2, p. 180-195, 2011.

FERREIRA, D. F. Sisvar: a computer statistical analysis system. Ciência e Agrotecnologia, Lavras, v. 35, n. 6, p. 1039-1042, 2011.

FORNAZIER, A.; WAQUIL, P. D. A produção integrada de frutas como um mecanismo de menor impacto ao meio ambiente. Cadernos de Ciência \& Tecnologia, Brasília, DF, v. 28, n. 1, p. 341-365, 2011.

GALEGO, L. G. C.; CARETO, C. M. A. Scenario of the spread of the invasive species Zaprionus indianus Gupta,
1970 (Diptera, Drosophilidae) in Brazil. Genetics and Molecular Biology, Ribeirão Preto, v. 33, n. 4, p. 767773, 2010.

GOMEZ, K. A.; GOMEZ, A. A. Statistical procedures for agricultural research. New York: International Rice Research Institute, 1984.

HELDWEIN, A. B.; BURIOL, A. G.; STRECK, N. A. O clima de Santa Maria. Ciência \& Ambiente, Santa Maria, v. 38, n. 1, p. 43-58, 2009.

LINDE, K. V. D. Zaprionus indianus: species identification and taxonomic position. Drosophila Information Service, Norman, v. 93, n. 1, p. 95-98, 2010.

MALO, E. A. et al. Attraction of the West Indian fruit fly to mango fruit volatiles. Entomologia Experimentalis et Applicata, Amsterdam, v. 142, n. 1, p. 45-52, 2012.

MATA, R. A. et al. Invasive and flexible: niche shift in the drosophilid Zaprionus indianus (Insecta, Diptera). Biological Invasions, Dordrecht, v. 12, n. 5, p. 1231-1249, 2010.

MAZOR, M. Competitiveness of fertilizers with proteinaceous baits applied in Mediterranean fruit fly, Ceratitis capitata Wied. (Diptera: Tephritidae) control. Crop Protection, Amsterdam, v. 28, n. 4, p. 314-319, 2009.

PASINI, M. P. B.; LINK, D. Efficiency of different traps to capture Zaprionus indianus (Diptera: Drosophilidae) in fig orchard in Santa Maria county, Rio Grande do Sul State, Brazil. International Research Journal of Agricultural Science and Soil Science, Sapele, v. 1, n. 8, p. 349-354, 2011.

PASINI, M. P. B.; LINK, D.; FRONZA, D. Eficácia de atrativos alimentares na captura de Zaprionus indianus (Gupta) em pomar de figo em Santa Maria - RS. Entomo Brasilis, Vassouras, v. 4, n. 2, p. 56-60, 2011a.

PASINI, M. P. B.; LINK, D.; SCHAICH, G. Attractive solutions efficiency in capturing Zaprionus indianus Gupta, 1970 (Diptera: Drosophilidae) in Ficus carica L. (Moraceae) orchard in Santa Maria, Rio Grande do Sul, Brazil. Entomotropica, Maracay, v. 26, n. 3, p. 107-116, 2011b.

PASINI, M. P. B.; LINK, D.; FRONZA, D. Eficiência de atrativos alimentares no monitoramento de Zaprionus indianus (Diptera: Drosophilidae) em pomar de goiaba. Revista Brasileira de Agroecologia, Cruz Alta, v. 7, n. 1, p. 152-159, 2012.

PIRES, D. J.; BELO, M.; BARBOSA, J. C. Life history estimatives in two geographic strains of Zaprionus indianus Gupta, 1970 (Diptera: Drosophilidae). Biodiversidade, Rondonópolis, v. 7, n. 1, p. 42-55, 2008. 
RAGA, A.; SOUZA FILHO, M. F. Captura de Zaprionus indianus (Gupta) (Dip: Drosophilidae) em frascos de plástico com iscas alimentares na cultura do figo. Revista de Agricultura, Piracicaba, v. 78, n. 3, p. 323-329, 2003.

RAGA, A. et al. Eficácia de atrativos alimentares na captura de moscas-das-frutas em pomar de citros. Bragantia, Campinas, v. 65, n. 2, p. 337-345, 2006.

SCOZ, P. L. et al. Avaliação de atrativos alimentares e armadilhas para o monitoramento de Anastrepha fraterculus (Wiedmann, 1830) (Diptera: Tephritidae) na cultura do pessegueiro (Prunus persicae (L.) Batsh). Idesia, Arica, v. 24, n. 2, p. 7-13, 2006.

SILVA, A. C. et al. Trocas gasosas e ciclo fotossintético da figueira 'Roxo de Valinhos'. Ciência Rural, Santa Maria, v. 40, n. 6 , p. 1270-1276, 2010.

SILVA, A. C. et al. Crescimento de figueira sob diferentes condições de cultivo. Pesquisa Agropecuária Tropical, Goiânia, v. 41, n. 4, p. 539-551, 2011.
STEIN, C. P.; TEIXEIRA, E. P.; NOVO, J. P. S. Aspectos biológicos da mosca do figo, Zaprionus indianus Gupta, 1970 (Diptera: Drosophilidae). Entomotropica, Maracay, v. 18, n. 3, p. 219-221, 2003.

TEIXEIRA, R. et al. Controle de pragas e doenças, maturação e qualidade de maçãs 'imperial gala' submetidas ao ensacamento. Revista Brassileira de Fruticultura, Jaboticabal, v. 33, n. 2, p. 394-401, 2011.

VILELA, C. R.; TEIXEIRA, E. P.; STEIN, C. P. Mosca-africana-do-figo, Zaprionus indianus (Diptera: Drosophilidae). In: VILELA, E. F.; ZUCHI, R. A.; CANTOR, F. Pragas introduzidas no Brasil. Ribeirão Preto: Holos, 2000. p. 48-52.

YASSIN, A. E.; DAVID, J. R. Revision of the afrotropical species of Zaprionus (Diptera, Drosophilidae), with descriptions of two new species and notes on internal reproductive structures and immature stages. ZooKeys, Bethesda, v. 51, n. 1, p. 33-72, 2010. 